\title{
GESTIÓN DE EXPATRIADOS: UN RETO PARA LAS EMPRESAS NACIONALES
}

Pedro L. Tito Huamani (")

E-mail: pedrotito18@hotmail.com

\section{RESUMEN}

El presente trabajo se refiere al tratamiento que las empresas dan -en términos de gestión humana- a sus directivos, funcionarios o profesionales altamente especializados, que mantienen por conveniencia en sus filiales en el extranjero.

La internacionalización de la empresa es una decisión de trascendencia que, en esencia, refleja su nivel de crecimiento y desarrollo en un contexto de productividad y competitividad no solo en el plano nacional sino, fundamentalmente, en el plano internacional. Dicha decisión lleva implícitas otras decisiones colaterales como definir quién o quiénes serán los encargados de gestionar la filial o filiales.

Cuando la empresa pasa de la exportación a la internacionalización vía filial o joint venture, le interesa sobremanera garantizar el éxito de sus operaciones, desde sus inicios, con la gestión de un funcionario de su entera confianza. Esta preocupación de gestión exitosa, así como la seguridad de su control, es lo que lleva a apostar por trabajadores nacionales que se encargarán de gestionar la filial o sucursal en otros países.

A este grupo de directivos/ funcionarios se les conoce como expatriados, de modo que gestionar desde la óptica internacional a tales trabajadores se convierte en todo un reto para las oficinas centrales de personal o recursos humanos de las empresas. En América Latina y en particular en el Perú, el tratamiento que se hace a este grupo de trabajadores es toda una novedad, dado que son muy pocas las organizaciones que se encuentran en esta fase: Ajegroup, Grupo Gloria, Grupo Crédito, entre otros.

Al respecto, la teoría hace referencia que las empresas exitosas en ambientes globales han puesto mucho énfasis en su talento humano como uno de los factores claves del éxito de sus procesos de internacionalización.

La mentalidad global de una compañía se afianza cuando todos los colaboradores piensan en el mundo como su mercado natural. Esta actitud mental influye definitivamente en los procesos de producción, comercial y en la eficiencia de los procesos operativos. Es así que la internacionalización

(") Magíster en Gestión Empresarial y Licenciado en Administración. Docente Investigador de la UNMSM. Docente de Postgrado UNMSM y Profesor visitante en las Escuelas de Postgrado de las universidades UNP (Piura), UNT (Trujillo), UNLG (Ica), UNA (Puno) y UNSCH (Ayacucho). Consultor en Gestión Humana y Comportamiento Organizacional. 
introduce y requiere de una nueva actitud de su talento humano; fundamentalmente en aquellos que tienen la responsabilidad de dirigir las filiales en el extranjero.

En la primera parte del presente trabajo revisamos la amplia teoría relacionada a la Gestión de Expatriados, analizando los pro y contras de esta decisión en el contexto de las culturas propias de los países de destino. De la misma forma, tratamos de demostrar la importancia que tiene para las empresas la gestión de su personal expatriado en los países de destino, describiendo lo que ocurre a nivel América Latina, y en particular a nivel del Perú.

En la parte de contrastación o evidencia empírica comentamos la experiencia exitosa de internacionalización de Ajegroup (Grupo Añaños), una empresa que nace en las entrañas de una ciudad provinciana (Huamanga) y que es en la actualidad una referencia obligada en el proceso de internacionalización de las empresas nacionales.

Finalmente, creemos que la internacionalización y dentro de ella la gestión de trabajadores expatriados es un fenómeno que habrán de experimentar muchas empresas nacionales, en el contexto de la vigencia de los Tratados del Libre Comercio (TLC) suscritos entre nuestro país y Estados Unidos, la Comunidad Europea, China, Chile, etc. En esta parte será importante continuar analizando las experiencias de algunos países vecinos que tienen mayor experiencia en el tema, como México, Chile, Brasil, entre otros.

Palabras clave: Gestión Humana, Gestión de Expatriados, Crecimiento Organizacional, Internacionalización.

\section{ABSTRACT}

This paper refers to treatment that companies give "in terms of human management" to its directors, officers or highly skilled professionals, who have for convenience in their subsidiaries abroad.

The internationalization of the firm is a decision of significance, which essentially reflects the level of growth and development, is understood in the context of productivity and competitiveness not only nationally, but primarily at the international level. This decision implies decisions other side, as that of defining who or who will be responsible for managing the subsidiary or subsidiaries.

When the firm moves from the export of internationalization, via subsidiary or joint venture, will be very interested with an official guarantee of their entire confidence, the success of its operations since its inception. This concern of successful management and security of your control, is what workers have to bet on national will manage the subsidiary or branch in other countries.

This group of Directors/Officers are known as expatriates. So managed from the international perspective to such workers, it becomes a challenge for headquarters personnel or human resources of enterprises. In Latin America and particularly in Peru, the treatment that makes this group of workers is a novelty, given that very few of them are in this phase: Ajegroup, Grupo Gloria, Group Credit, being other.

In this regard, the theory refers to the successful companies in global environments have placed considerable emphasis on its human talent, as one of the key success factors of their internationalization processes.

The global mindset is taking hold in a company where all employees feel in the world as a natural market. This mental attitude definitely affects production processes, business and efficiency of operational processes. Thus, globalization introduces and requires a new attitude of its human talent, particularly in those who have responsibility for directing the foreign subsidiaries.

The first part of this paper we review the broad theory related to the Management of Expatriates, analyzing the pros and cons of this decision in the context of the cultures of the countries of destination. Similarly, we try to demonstrate the importance for enterprises, management of its expatriate staff in destination countries, describing what is happening at Latin America, particularly Peru level.

In the contrastive or empirical evidence discussed the successful experience of internationalization Ajegroup (Group Añaños), a company born in the bowels of a provincial city (Huamanga), which is now a reference in the internationalization process domestic firms.

Finally, we believe that internationalization and within the management of expatriate workers, is a 
phenomenon that must undergo many domestic firms, in the context of the validity of the Treaties of Free Trade Agreements (FTAs) signed between our country and the United States America (USA), the European Community (EC), China, Chile, etc. This part will be important to continue analyzing the experiences of some neighboring countries, who have more experience than us, such as Mexico, Chile, Brazil, among others.

Key words: Human Management, Expatriate Management, Organizational Growth, Internationalization.

\section{PROBLEMA EN LA INVESTIGACIÓN}

El fenómeno de la expatriación está ocasionando numerosos problemas de gestión humana debido a la inexperiencia o imprevisión de las empresas. El costo en capital económico y humano que ello supone ha puesto de manifiesto la necesidad de profesionalizar al máximo el proceso de expatriación.

Además, las empresas han cobrado conciencia de su importancia para el éxito de la estrategia de internacionalización, lo que ha despertado un interés creciente por conocer las particularidades de su gestión.

El traslado de profesionales a otros países plantea un reto de múltiples implicaciones, por lo que debe afrontarse con una visión global que dé coherencia a las políticas de actuación. Para ello, es importante identificar las características del proyecto empresarial y los objetivos que se pretenden alcanzar.

La gestión actual de los expatriados se centra básicamente, en el plano empresarial, en la eficacia, y en el plano económico, en el costo en que se incurre, dejando de lado los planos personal, profesional y familiar que están prácticamente fuera del proceso de decisión.

La falta de una planificación previa de la trayectoria profesional de la persona, así como la escasa importancia que se le asigna a la dimensión familiar, acarrean importantes problemas que ponen en jaque el éxito final del proceso. Los expatriados se acostumbran a determinados beneficios económicos, sociales (prestigio y rango) y profesionales (autonomía y responsabilidad), cuya pérdida resulta difícil de asimilar al regresar a su país de origen.

Tras varios años de esfuerzo, y en ocasiones sacrificio, los repatriados tampoco comprenden cómo no se les recompensan o no se aprovechan los conocimientos que han adquirido. Cuando las empresas deciden por su internacionalización, en el plano de la gestión humana, surgen las siguientes preguntas:

- ¿Cuál es el perfil del profesional que garantizará un desenvolvimiento exitoso en el país de destino?

- ¿Qué problemas sociales, personales e incluso familiares acarreará a la empresa la expatriación de funcionarios?

- ¿Qué políticas y procesos de la gestión humana se deben reestructurar en la empresa para gestionar al personal expatriado?

\section{IMPORTANCIA DE LA INVESTIGACIÓN}

Investigar la gestión de los expatriados es importante porque de ella dependen las posibilidades que tienen las empresas de consolidar su ingreso y posicionamiento en el mercado extranjero.

En un contexto de globalización y competencia frontal, el tener a funcionarios connacionales de nuestra confianza que garanticen el cumplimiento de objetivos y metas, y den seguridad para el control de la filial (país de destino), puede ser la ventaja diferencial para las empresas, que en el caso nuestro tienen poca experiencia en el proceso de internacionalización.

Este interés adquiere mayor importancia por el hecho de que nuestro país tiene un Convenio vigente de Tratado de Libre Comercio (TLC) con el país que tiene el mayor mercado en el mundo que es Estados Unidos, con la Comunidad Europea, China y con Chile, lo que conllevará a las empresas a interesarse en cómo se gestiona a este grupo de profesionales que permanecen en otros países por un determinado tiempo.

\section{OBJETIVOS DE LA INVESTIGACIÓN}

Se ha creído oportuno plantearse los siguientes objetivos de la investigación, que se precisan seguidamente: 
- Demostrar la importancia que tiene para las empresas la gestión de su personal expatriado en los países de destino.

- Describir que a nivel América Latina, y en particular a nivel del Perú, la expatriación de personal es un fenómeno nuevo que las empresas deben priorizar si desean tener éxito en el mercado internacional.

- Comentar la experiencia de Ajegroup en el proceso de expatriación.

\section{MARCO DE REFERENCIA}

A medida que las empresas se internacionalizan, las prácticas de gestión humana adquieren una perspectiva global para facilitar a las empresas obtener su ventaja competitiva. Los directivos deben encontrar una fórmula para seleccionar, conservar, promocionar, recompensar y formar a los trabajadores, para que ayuden a superar el desafío de la globalización

\section{Etapas de la internacionalización}

La mayoría de los académicos coinciden en que en el proceso de crecimiento y desarrollo, las empresas transitan por las siguientes etapas que a continuación se mencionan:

a) Etapa 1: actividades nacionales. El mercado de la empresa es exclusivamente nacional. En cualquier país, la mayoría de las empresas se ubican en esta etapa. El personal, la formación y las remuneraciones en este tipo de empresas dependen directamente de factores locales y/o nacionales. Sus plantas de fabricación se encuentran localizadas en ciudades o regiones nacionales.

b) Etapa 2: actividades de exportación. La empresa amplía su mercado para incluir a otros países del extranjero, pero mantiene las instalaciones productivas dentro de las fronteras nacionales. En esta etapa, las prácticas de gestión de recursos humanos deben facilitar la exportación de productos de la empresa mediante incentivos a directivos, formación adecuada y estrategias de personal que se centran en la demanda de los consumidores internacionales.

c) Etapa 3: filiales o joint venture. La empresa traslada físicamente algunas de sus operaciones al país de destino. Estas instalaciones se utilizan fundamentalmente para ensamblar componentes, aunque puede haber cierta producción en el exterior. Las filiales o subsidiarias extranjeras tienden a estar bajo el férreo control de la sede de la empresa en esta etapa, y una gran proporción de los altos directivos son expatriados. Las prácticas de gestión de recursos humanos en esta etapa se centran en la selección, formación y remuneración de los expatriados, así como en el desarrollo de las políticas de personal de los empleados locales en las instalaciones del país de origen.

d) Etapa 4: operaciones multinacionales. La empresa se convierte en una auténtica empresa multinacional (EMN), con instalaciones productivas y de ensamblaje en varios países y regiones del mundo. Aunque suele haber cierta descentralización en la toma de decisiones en las empresas que se encuentran en esta etapa, muchas decisiones de personal relativas a las sucursales extranjeras siguen tomándose en la sede principal de la empresa, normalmente por un departamento de personal internacional. Además, las actividades en el extranjero siguen siendo dirigidas por trabajadores expatriados. Las prácticas de gestión de recursos humanos de estas empresas son bastante complejas porque deben ocuparse de un gran número de expatriados y sus familias, de las asignaciones en el extranjero y de los diversos grupos étnicos y culturales que existen en los múltiples países donde opera.

e) Etapa 5: actividades transnacionales. Las empresas que alcanzan esta etapa suelen denominarse transnacionales porque les queda muy poca relación exclusiva con su país de origen, así como vínculos débiles con cualquier país en concreto. Las operaciones están muy descentralizadas y cada unidad empresarial es libre de tomar decisiones sobre el personal, con muy poco control de la sede principal. El directorio suele estar compuesto por personas de distintas nacionalidades y la empresa intenta desarrollar directivos que se consideren ciudadanos del mundo. Estas empresas contratan empleados libremente en cualquier país. Las prácticas de gestión de recursos humanos de las empresas que se ubican en esta etapa están diseñadas para combinar individuos de distinta procedencia y crear una identidad empresarial compartida (más que una nacional) y una visión común. 


\section{Decisiones sobre la dirección de la empresa en el extranjero}

Cuando la empresa pasa de la etapa de exportación (segunda etapa) a la etapa en que abre una sucursal extranjera (tercera etapa) -ya sea con una sucursal de propiedad exclusiva (la sucursal extranjera pertenece totalmente a la sede principal de la empresa) o una joint venture (parte de la sucursal en el extranjero pertenece a una entidad del país de acogida, otra empresa, un consorcio de empresas, un individuo o el gobierno), debe decidir quién va ser el responsable de dirigir la unidad. Esta decisión es importante porque, en la mayoría de los casos, la inversión necesaria para la fábrica y los equipos es enorme, y el éxito de la empresa en el extranjero depende en gran medida de quién está en el cargo.
Así existen los siguientes planteamientos para dirigir una filial en el extranjero (ver resumen en el Cuadro $\mathrm{N}^{\circ} 1$ ):

a) Etnocéntrico. La Alta Dirección y otros puestos claves son ocupados por personas del país de origen. La empresa utiliza un gran grupo de directivos expatriados. La gestión de recursos humanos internacional participa en el reclutamiento, desarrollo y la remuneración en todo el mundo y son directamente responsables ante el directivo más alto de la corporación. El directivo es un antiguo expatriado que pasa gran parte de su tiempo supervisando las actividades internacionales. Este tipo de políticas tiene el problema que en ciertas culturas generan rechazos hacia la gerencia y le quitan legitimidad a las decisiones tomadas.

Cuadro $\mathrm{N}^{\circ} 1$

Planteamientos para dirigir una filial en el extranjero - Resumen

\begin{tabular}{|c|c|c|c|c|}
\hline $\begin{array}{l}\text { ASPECTOS DE LA } \\
\text { EMPRESA }\end{array}$ & ETNOCÉNTRICA & POLICÉNTRICA & REGIOCÉNTRICA & GEOCÉNTRICA \\
\hline $\begin{array}{l}\text { Complejidad de la } \\
\text { organización }\end{array}$ & $\begin{array}{l}\text { Compleja en la casa } \\
\text { matriz, sencilla en sus } \\
\text { subsidiarias. }\end{array}$ & $\begin{array}{l}\text { Variada e indepen- } \\
\text { diente. }\end{array}$ & $\begin{array}{l}\text { Altamente independien- } \\
\text { te, fundamentada en } \\
\text { conceptos regionales. }\end{array}$ & $\begin{array}{l}\text { Crecientemente compleja y } \\
\text { altamente independiente, } \\
\text { fundamentada en conceptos } \\
\text { globales. }\end{array}$ \\
\hline $\begin{array}{c}\text { Autoridad y toma de } \\
\text { decisiones }\end{array}$ & $\begin{array}{c}\text { Alta en la oficina } \\
\text { central. }\end{array}$ & $\begin{array}{l}\text { Relativamente baja en } \\
\text { la oficina central. }\end{array}$ & $\begin{array}{l}\text { Alta en las oficinas } \\
\text { regionales y/o alta } \\
\text { colaboración entre } \\
\text { subsidiarias. }\end{array}$ & $\begin{array}{l}\text { Colaboración entre la oficina } \\
\text { principal y las subsidiarias } \\
\text { alrededor del mundo. }\end{array}$ \\
\hline Evaluación y control & $\begin{array}{c}\text { Lineamientos estableci- } \\
\text { dos por la casa matriz } \\
\text { aplicados al personal } \\
\text { y a la ejecución de las } \\
\text { operaciones. }\end{array}$ & $\begin{array}{l}\text { Establecida local- } \\
\text { mente. }\end{array}$ & $\begin{array}{l}\text { Determinada regional- } \\
\text { mente. }\end{array}$ & $\begin{array}{l}\text { Recompensas con carácter } \\
\text { local y universal. }\end{array}$ \\
\hline $\begin{array}{l}\text { Recompensas y casti- } \\
\text { gos, incentivos }\end{array}$ & $\begin{array}{l}\text { Altos en la oficina } \\
\text { central, bajos en las } \\
\text { oficinas subsidiarias. }\end{array}$ & \begin{tabular}{|c} 
Amplia variedad, pue- \\
de tener altos o bajos \\
incentivos para el \\
desempeño regional.
\end{tabular} & $\begin{array}{c}\text { Incentivos a la contribu- } \\
\text { ción hacia los objetivos } \\
\text { regionales. }\end{array}$ & $\begin{array}{l}\text { Recompensa a los ejecutivos } \\
\text { internacionales y locales por } \\
\text { el cumplimiento de metas } \\
\text { regionales y globales. }\end{array}$ \\
\hline $\begin{array}{l}\text { Comunicación, flujo } \\
\text { de información }\end{array}$ & $\begin{array}{c}\text { Alto volumen de órde- } \\
\text { nes, disposiciones y } \\
\text { notificaciones hacia las } \\
\text { subsidiarias. }\end{array}$ & $\begin{array}{c}\text { Reducida desde y } \\
\text { hacia la oficina princi- } \\
\text { pal, pequeña entre las } \\
\text { subsidiarias. }\end{array}$ & $\begin{array}{l}\text { Pequeña desde y hacia } \\
\text { la oficina central, puede } \\
\text { ser alta desde y hacia } \\
\text { las oficinas regionales y } \\
\text { los países. }\end{array}$ & $\begin{array}{c}\text { En ambas direcciones entre } \\
\text { las subsidiarias alrededor del } \\
\text { mundo. }\end{array}$ \\
\hline Identidad geográfica & $\begin{array}{c}\text { Nacionalidad del pro- } \\
\text { pietario. }\end{array}$ & $\begin{array}{c}\text { Nacionalidad del país } \\
\text { anfitrión. }\end{array}$ & Compañía regional. & $\begin{array}{l}\text { Compañía realmente global, } \\
\text { pero con reconocimiento de } \\
\text { los intereses locales. }\end{array}$ \\
\hline $\begin{array}{c}\text { Reclutamiento de } \\
\text { personal, aprovisio- } \\
\text { namiento y desa- } \\
\text { rrollo }\end{array}$ & $\begin{array}{l}\text { Nacionales del país de } \\
\text { origen ocupando las } \\
\text { posiciones clave alre- } \\
\text { dedor del mundo. }\end{array}$ & $\begin{array}{l}\text { Nacionales del país } \\
\text { anfitrión ocupando } \\
\text { las posiciones clave } \\
\text { en su país. }\end{array}$ & $\begin{array}{l}\text { Individuos de la región } \\
\text { ocupando los altos car- } \\
\text { gos dentro de la región. }\end{array}$ & $\begin{array}{l}\text { Los mejores ejecutivos de } \\
\text { todo el mundo ocupando los } \\
\text { más altos cargos en cual- } \\
\text { quier parte. }\end{array}$ \\
\hline
\end{tabular}


Igualmente los gerentes del país de origen generalmente pretenden adaptar la cultura a su estilo gerencial y no adaptar su estilo de gerencia a la cultura local; sin embargo esta política es muy efectiva en países donde no hay personal calificado para llenar las posiciones gerenciales.

b) Policéntrico. Las filiales internacionales son dirigidas y tienen trabajadores del país local o de destino. Tiene la ventaja de una mayor motivación de la fuerza de trabajo y una mayor legitimidad de las decisiones gerenciales, pero no siempre es fácil ni barato encontrar personas perfectas para estos puestos. Tiene la ventaja de que el personal de la gerencia ya está familiarizado con la cultura donde se desarrollarán las operaciones de la empresa, minimizando así los costos de introducción en una nueva cultura.

c) Geocéntrico. Se concede deliberadamente poca importancia a la nacionalidad y la empresa busca activamente la gente más adecuada para los puestos clave en todo el mundo. Son propios de las empresas transnacionales. Es básicamente la máxima expresión de la meritocracia, los altos ejecutivos son rotados por todo el mundo. Esto tiene muchas ventajas, especialmente al tener gerentes de muchos países gestionando las operaciones de la empresa en cualquier país; sin embargo, tiene problemas en cuanto al shock cultural de los funcionarios y la dificultad de adaptar las estrategias gerenciales a las diferentes culturas.

d) Regiocéntrico. Es cuando las empresas deciden por la dirección de la filial con personal que pertenece a la misma región geográfica. Por ejemplo: una vacante que se presente en el Perú, siguiendo este criterio, puede ser cubierta por un ejecutivo que proviene de Argentina, Chile u otro país de América Latina.

\section{Razones que motivan a las empresas a decidirse por los trabajadores expatriados}

La mayoría de las empresas decide utilizar a los trabajadores expatriados para ocupar los puestos claves en la organización como directivos, ejecutivos, profesionales de alto nivel y especialistas técnicos, aunque tener a trabajadores expatriados es relativamente caro en comparación a un trabajador local. Por tanto la empresa apostará por un trabajador expatriado en los siguientes casos:
- No existe la cualificación necesaria en el país de destino. Esto es muy probable en empresas que tienen actividades en países como el nuestro.

- Una parte importante de la estrategia empresarial general de la empresa es la creación de una visión global. Algunas empresas prefieren que sus filiales sean parte de una red internacional con una identidad empresarial compartida. Cuando este es el caso, los expatriados se utilizan para unir las filiales internacionales de la organización.

- Las unidades internacionales y las actividades nacionales son muy interdependientes. En algunos casos, el proceso productivo exige que todas las divisiones de la empresa, tanto nacionales como internacionales, colaboren estrechamente. Esto es particularmente necesario cuando el producto de una unidad empresarial es necesario como materia prima de otra unidad de la misma empresa.

- La situación política es inestable. Las empresas tienden a utilizar expatriados para los puestos de Alta Dirección cuando el riesgo de intervención gubernamental de un negocio es elevado, dado que existe agitación política, actual o potencial en el país, o cuando existe amenaza terrorista, y cuando ha habido un historial reciente de revolución social en el país.

- Existen importantes diferencias culturales entre el país de origen y el país de destino. Cuanto más diferente sea la cultura donde se encuentra la filial respecto a la cultura de la sede principal (en cuanto a idioma, religión, costumbres, etc.), más importante será nombrar a expatriados que puedan actuar de intérpretes y enlace entre las dos culturas. Puesto que esto requiere disponer de una gran sensibilidad ante otras culturas, la empresa transnacional tiene que seleccionar y formar cuidadosamente a los individuos que van a ocupar estos puestos. Esto puede exigir una cuidadosa planificación de la carrera profesional.

Las ventajas o desventajas que ofrecen a la empresa el tener trabajadores expatriados son los siguientes:

\section{a) Ventajas:}

- Analogía cultural con la empresa matriz que garantiza la transferencia de prácticas empresariales/ directivas. 
- Permite un control más estrecho y una mejor coordinación de las filiales internacionales.

- Ofrece a los trabajadores una orientación multinacional gracias a la experiencia de la empresa matriz.

- Crea un conjunto de ejecutivos con experiencia internacional.

- Puede no haber una calificación disponible en el país de destino, como para obtener todo el valor que ofrecen los expatriados.

- Ofrece una perspectiva global más amplia.

\section{b) Desventajas:}

- Crea problemas de adaptación al entorno y a la cultura extranjera.

- Aumenta la "foraneidad" de la filial.

- Puede implicar elevados costos salariales, de transferencia y otros.

- Puede provocar problemas personales y familiares.

- Tiene un efecto negativo sobre la moral y la motivación de los directivos nacionales.

- Su contratación puede estar sujeta a las restricciones del gobierno del país de destino.

\section{Ventajas y desventajas de utilizar trabajadores locales}

De la misma forma, la decisión de apostar por un directivo/ funcionario local pasa necesariamente por tener que analizar las ventajas o desventajas del directivo local para la empresa. Así tenemos:

\section{a) Ventajas:}

- Menores costos laborales.

- Es un indicador de confianza en los profesionales del país de destino.

- Mejora la aceptación de la empresa en la comunidad.

- Maximiza el número de opciones disponibles en el entorno local.

- Permite obtener el reconocimiento de la empresa como un participante legítimo en la economía local.
- Representa eficazmente las consideraciones y restricciones locales en el proceso de toma de decisiones.

- Mayor comprensión de las condiciones locales.

b) Desventajas:

- Dificulta el equilibrio entre las exigencias locales y las prioridades globales.

- Suele provocar que se pospongan las decisiones locales difíciles (por ejemplo, la reducción de la planilla) hasta que se hacen inevitables, cuando es más difícil, costoso y doloroso tomar decisiones que si se hubieran hecho antes.

- Puede dificultar el reclutamiento de personal cualificado.

- Puede reducir la cantidad de control ejercido por la sede principal.

\section{La gestión humana en el contexto de América Latina}

La administración en América Latina está definida por rasgos culturales que una vez identificados podrían explicar muchas de las formas y prácticas del trabajo en la región. Se concibe a las organizaciones como que están inmersas en tradiciones históricas, a pesar de que las fuerzas de la globalización están provocando cambios profundos en la región.

- La distancia de poder está representada de diferentes maneras dentro de las organizaciones latinoamericanas.

- Liderazgo paternalista benevolente. El concepto de familia se convierte en una metáfora para dirigir las organizaciones.

- Las relaciones sociales en el centro laboral se manifiestan a través de contacto personal (alto contenido emocional); lealtad al grupo y costumbres populares como tradiciones religiosas $\mathrm{y}$ festividades sociales.

a) Sistema de trabajo. La cultura latinoamericana tiende a favorecer la implantación de equipos de trabajo. Esto se debe a que valoramos las relaciones sociales que se manifiestan a través de la comunicación personal y la empatía entre colegas. Así mismo, valoramos de las empresas sus estructuras centralizadas y jerarquías bien definidas. Sin embargo, la tendencia en 
la gestión moderna va por el lado de impulsar la descentralización e incluso promover un proceso de democratización de la autoridad.

La toma de decisiones compartida con la gerencia también ilustra el modelo híbrido porque los latinoamericanos desean evitar el conflicto y la confrontación, no solamente con los superiores sino también con sus compañeros; por tanto aceptan un estilo de liderazgo directivo que medie entre las partes en conflicto, un estilo que quizás se necesite durante la dinámica del trabajo en equipo.

b) Rol de la familia. La familia es muy importante para los trabajadores en América Latina. Las decisiones que se toman en materia laboral están condicionadas por la búsqueda del bienestar de la familia. Esta política asume que la confianza, lealtad y responsabilidad son atributos formados en el núcleo familiar, muy reconocidos por las empresas.

c) Recompensas y reconocimientos. Como la familia es el núcleo central en la vida del trabajador de América Latina, el trabajo debe coadyuvar al bienestar material de la familia. La mayoría de las legislaciones laborales en la región están orientadas a que el trabajador obtenga los medios económicos para enfrentar las crisis económicas recurrentes. Así, las retribuciones están dirigidas a garantizar la estabilidad económica de la familia, más que a promover el desarrollo individual del trabajador.

El trabajo en esta parte del continente también satisface las necesidades sociales del trabajador. El reconocimiento social se convierte en un importante factor motivador para fomentar la cooperación y lealtad, rasgos importantes en una sociedad colectiva.

La lealtad de los trabajadores se reconoce socialmente por medio de ceremonias que recompensan la antiguiedad en el empleo. La antiguiedad en nuestra región es importante y muchos de los beneficios del trabajo dependen de ésta.

d) Reclutamiento y selección. Se basa generalmente en rasgos de la personalidad y características físicas de la persona:

- Paternalismo benevolente: contratación de familiares o amigos cercanos al trabajador.
- Conflicto y confrontación: se contratan personas que contribuyen al buen clima laboral.

- Distancia social: "Techo de Cristal" basado en apariencia física y contactos sociales.

- Relaciones sociales: la principal fuente de nuevos empleados en muchas empresas de América Latina se encuentra en los mismos trabajadores.

e) Entrenamiento y desarrollo. Las empresas experimentan conflictos encontrados. Por un lado, necesitan entrenar a la fuerza laboral en prácticas modernas de administración tales como equipos autodirigidos $y$, por otro lado, saben que no todas las prácticas encajan con la idiosincrasia regional y pueden ser rechazadas o modificadas de acuerdo a la mentalidad y experiencia latinoamericana.

Además de desarrollar las habilidades de los trabajadores para sistemas de trabajo moderno, las empresas latinoamericanas con mínimo presupuesto deben ofrecer entrenamiento técnico e incluso educación básica formal, dado que es un obstáculo serio en la región la falta de conocimiento técnico, la educación formal, y la carencia de habilidades analíticas y de comunicación.

\section{LA EXPERIENCIA DE EXPATRIACIÓN EN EMPRESAS LOCALES: EL CASO DE AJEGROUP.}

Industrias Añaños es una empresa familiar fundada en 1988 en el departamento de Ayacucho, con el objetivo de dedicarse a la elaboración y distribución de bebidas gaseosas y aguas de mesa. Luego de expandirse exitosamente por el interior del país, en 1997 la empresa decidió entrar a competir al mercado limeño empleando una exitosa estrategia de calidad a bajos precios.

La empresa cuenta con plantas ubicadas estratégicamente en Huamanga, Huancayo, Tarapoto, Iquitos, Pucallpa, Lima, Huaura, Sullana, Trujillo y Arequipa para abastecer el mercado peruano, y otras tantas instaladas en diversos países como Venezuela, Ecuador, México, Costa Rica, Nicaragua, Guatemala, Honduras y Tailandia. La corporación Ajegroup emplea en el Perú a 8,500 trabajadores en forma directa e indirecta.

Las marcas que maneja la empresa son variadas y se dirigen a diversos segmentos:

- Gaseosas: Kola Real, Sabor de Oro y Big Cola. 
- Agua de mesa: Cielo.

- Energizante: Sporade.

- Esencia de frutas: Pulp.

- Cervezas: las marcas Franca y Caral, últimamente.

\section{Estrategias comerciales}

a) La bebida del precio justo. Para el lanzamiento de Kola Real al mercado limeño, la empresa utilizó una ingeniosa estrategia de posicionamiento: "La Bebida del Precio Justo", con lo que creció el mercado por la incorporación de consumidores de los sectores C, D y E. La ingeniosa estrategia de colocar a Kola Real como la bebida del precio justo y no como la más barata, hizo alusión a que las demás bebidas colas eran caras y no puso en duda la calidad del producto, mostrando en sus comerciales las instalaciones de sus plantas, proyectando una imagen de limpieza.

b) Concentración en un sector con amplio potencial de crecimiento. Ajegroup decidió no competir frontalmente con las grandes embotelladoras al concentrarse en mercados con amplio potencial de crecimiento como los extractos socioeconómicos C, D y E, público objetivo de la empresa, que representa el 85\% de la población total urbana, induciendo al consumo a sectores que antes no lo hacían.

c) Ofrecer calidad a bajos precios. La empresa no paga royalties por uso de marcas como muchas de las empresas más grandes con las que compite, lo cual les permite ofrecer a menor precio presentaciones de mayor litraje que la competencia. Adicionalmente, el uso intensivo de envases PET posibilitó el transporte de gaseosas a grandes distancias, cubriendo mercados poco accesibles para las gaseosas con envase de vidrio.

d) Internacionalización. En 1999, Ajegroup decide internacionalizar su marca para no depender exclusivamente del mercado peruano, previendo la dura respuesta de las demás embotelladoras para defender su participación en el mercado local. Así, la empresa ingresa al mercado venezolano de bebidas gaseosas fundamentándose en el factor climático (temperaturas altas durante la mayor parte del año) y en el hecho de que este mercado tiene el doble del tamaño que el peruano. Cabe mencionar que antes de la introducción en Venezuela de Kola Real en envase plástico no retornable, las gaseosas en ese país se distribuían solo en envases de vidrio. La estrategia permitió a Ajegroup capturar el $12 \%$ del mercado, equivalente al 30\% del mercado peruano.

En el año 2001, Industrias Añaños decidió ingresar al mercado ecuatoriano; en una primera fase mediante la exportación de las marcas Kola Real y Sabor de Oro a través de su embotelladora de Sullana (ubicada al norte del Perú), para luego fortalecer su presencia con la instalación de una planta en la ciudad de Machala.

En el año 2002 la empresa se embarcó en lo que podría ser su mayor reto: ingresar al mercado mexicano, el cual registra el mayor consumo de gaseosas del mundo luego del norteamericano, para lo cual instaló una planta en Puebla, invirtiendo cerca de US\$ 7 millones. Con esta planta abastece a las ciudades de Puebla, Veracruz y Acapulco, esperando obtener en los siguientes años el 5\% del mercado, lo cual equivale al 69\% del mercado peruano.

Con esta internacionalización la empresa reduce su exposición en el mercado peruano y rompe con la estacionalidad de las ventas, considerando que el verano en México se presenta entre julio y agosto mientras que en Perú se presenta en los meses de diciembre a marzo.

e) Sistemas de distribución de microempresarios. Desde sus inicios Industrias Añaños siempre apostó por una red de distribución hecha por microempresarios quienes, con sus propios medios de transporte, se encargan de distribuir el producto en las diferentes zonas asignadas, lo cual coadyuvó al rápido crecimiento de las ventas ya que se prescindió de invertir en una red de distribución propia. Actualmente la empresa llega a 180,000 puntos de venta en el Perú.

f) Oferta de diferentes sabores y presentaciones en sus envases PET desechables. Industria Añaños produce bebidas de tipo fantasía (Kola Real) para competir con marcas como Coca Cola, Pepsi Cola, Fanta, Sprite y Crush; y bebida amarilla (Sabor de Oro) para competir con la tradicional Inca Kola. Mediante esta estrategia, la empresa está en capacidad de hacer frente a las diversas marcas existentes en el merca- 
do por sabor y/o litraje a precios menores al emplear envases PET desechables.

\section{Capacidades claves de Industrias Añaños.}

Las capacidades clave de Ajegroup son las relacionadas a su estrategia de enfoque en los segmentos de mercado de menor poder adquisitivo, a través de productos de bajo precio y buena calidad. Se han distinguido dos capacidades clave, cada una se describe a continuación:

\section{a) Penetración a nuevos mercados y crecimiento rápido.}

(Ver Cuadro $\mathrm{N}^{\circ}$ )

b) Gestión de trabajadores expatriados. En su proceso de internacionalización, la Corporación Ajegroup ha venido apostando a que la responsabilidad de conducción de la empresa en cada país de destino recaiga en uno de los hermanos Añaños. De la misma forma, el equipo ejecutivo de primer nivel siempre ha sido confiado en cada país a connacionales nuestros. Esta decisión obedece a que la empresa quiere garantizar el cumplimiento de los objetivos y metas propuestas.

De la misma forma, interesa que en las filiales se ejecuten las políticas y directivas que ema- nan de la Alta Dirección de Ajegroup. Independientemente a ello, para garantizar en los colaboradores el proceso de internacionalización de la filosofía y cultura de la organización, la corporación desarrolla las siguientes líneas de acción:

- Capacitación y entrenamiento constante de los colaboradores de la compañía. Es política de la empresa mantener a sus trabajadores actualizados, para lo cual diseña periódicamente un plan de capacitación continua.

- Busca mantener colaboradores especializados en distintas áreas de la organización.

- Práctica de una relación afectiva con los colaboradores; dado que la empresa se gestiona bajo una concepción familiar, se transmite el sentido familiar en la compañía.

\section{CONCLUSIONES}

1. La internacionalización es parte del proceso de desarrollo de las empresas. Toda empresa que llega a cubrir el mercado nacional necesariamente debe pensar en dar el salto cualitativo de incursionar en el mercado internacional.

Cuadro $\mathrm{N}^{\circ} 2$

Ajegroup: Penetración y crecimiento

\begin{tabular}{|l|l|}
\hline \multicolumn{1}{|c|}{ Criterio } & \multicolumn{1}{c|}{ Aplicación } \\
\hline Es difícil de imitar. & $\begin{array}{l}\text { La evidencia de que esta capacidad es difícil de imitar está en la existencia de } \\
\text { varias marcas regionales que no han podido explotar el potencial de ventas que } \\
\text { existe en el mercado limeño. }\end{array}$ \\
\hline $\begin{array}{l}\text { Requiere de comunicación, involu- } \\
\text { cramiento y compromiso a través de } \\
\text { las "barreras" dentro de la organi- } \\
\text { zación. }\end{array}$ & $\begin{array}{l}\text { El desarrollo de esta capacidad es resultado del sacrificio realizado por los } \\
\text { propietarios, dado que se ha tenido limitado acceso al financiamiento externo. } \\
\text { Existe claridad a través de la organización que alcanzar las metas dentro de } \\
\text { un mercado es un proceso que requiere del aporte coordinado de todas las } \\
\text { funciones. }\end{array}$ \\
\hline $\begin{array}{l}\text { Ofrece potencial en una amplia } \\
\text { variedad de mercados. }\end{array}$ & $\begin{array}{l}\text { La empresa ha llevado el modelo de negocio a otros países con éxito, ganando } \\
\text { rápidamente participación de mercado. La aplicación de esta capacidad a otros } \\
\text { negocios es uno de los fines de su estrategia comercial. }\end{array}$ \\
\hline $\begin{array}{l}\text { Parte del capital intelectual de la } \\
\text { organización tiene que ver con su } \\
\text { comportamiento. }\end{array}$ & $\begin{array}{l}\text { El know-how de penetración y crecimiento con enfoque particular a los segmen- } \\
\text { tos C, D y E es propio de Industrias Añaños, a tal grado que ha podido desafiar } \\
\text { a marcas poderosas como Pepsi en Venezuela y Coca Cola en México como } \\
\text { ninguna otra ha podido. }\end{array}$ \\
\hline $\begin{array}{l}\text { Ofrece una contribución significati- } \\
\text { va al valor del producto que percibe } \\
\text { el cliente. }\end{array}$ & $\begin{array}{l}\text { El valor para el cliente está en haber satisfecho una demanda latente en seg- } \\
\text { mentos cuya capacidad económica no permite la compra de gaseosas de mayor } \\
\text { precio. }\end{array}$ \\
\hline
\end{tabular}


2. El mercado internacional plantea a las empresas desafíos relacionados con la productividad y competitividad.

3. Desde el punto de vista de la gestión humana, las empresas deben perfeccionar sus políticas y procesos para gestionar a los funcionarios expatriados.

4. Como se deduce del presente artículo, aprender a gestionar a los expatriados es un reto para las empresas de América Latina y en particular para las empresas peruanas, dado que son pocas las que se encuentran a ese nivel.

5. La gestión de expatriados por las empresas nacionales es un campo novedoso que requiere ser profundizado en la investigación, tomando como referencia las experiencias de grupos empresariales, tales como Ajegroup, Grupo Gloria, entre otros.

\section{BIBLIOGRAFÍA}

Chiavenato, Idalberto. (2003). Gestión del Talento Humano. Colombia, Editorial Mc Graw Hill.

Elvira, M. y Dávila, A. (2005). "Cultura y Administración de Recursos Humanos en América Latina”. En: Revista Universia Business Review - Actualidad Económica. Primer Trimestre 2005. España, Grupo Recoletos Comunicación.
Elvira, M. y Dávila, A. (2006). "Retos emergentes para la investigación de la administración de recursos humanos en América Latina”. En: Revista de Empresa. Enero - marzo 2006. Monterrey, Escuela de Graduados en Administración y Dirección de Empresas (ITESM).

Gómez-Mejía y otros. (2001). Dirección y Gestión de Recursos Humanos. Tercera Edición. Madrid, Pearson Educación, 728 págs.

Iacoviello, M y Zuvanic, L. (2007). "Desarrollo e Integración de la Gestión de Recursos Humanos en los estados latinoamericanos". En: Documentos y aportes en Administración Pública y Gestión Estatal. Vol. 6, enero 2007. Argentina, Universidad Nacional del Litoral.

Peña, I. y Hernández, F. (2007). "La integración de la Dirección de Recursos Humanos en el proceso de formulación de la estrategia como determinante de los resultados". En: Revista Universia Business Review - Actualidad Económica. Tercer Trimestre 2007. España, Unidad Editorial Sociedad de Revistas S.L.U.

\section{INTERNET}

Portal del Grupo Añaños Jerí. En: http://www. Ajegroup.com

Portal del Ministerio de Trabajo y Promoción del Empleo. En: http://www.mintra.gob.pe 